\title{
Orthogonal basis for the optical transfer function
}

\author{
Chelo Ferreira ${ }^{1}$, José L. López ${ }^{2}$, Rafael Navarro ${ }^{3,}$, and Ester Pérez Sinusía ${ }^{1}$ \\ ${ }^{1}$ Dpto. de Matemática Aplicada \& IUMA, Universidad de Zaragoza, 50009 Zaragoza, Spain \\ ${ }^{2}$ Dpto. de Ingeniería Matemática e Informática, Universidad Pública de Navarra, 31006 Pamplona, Spain \\ ${ }^{3}$ ICMA, Consejo Superior de Investigaciones Científicas \& Universidad de Zaragoza, 50009 Zaragoza, Spain \\ *Corresponding author: rafaelnb@unizar.es
}

Compiled September 19, 2016

\begin{abstract}
We propose systems of orthogonal functions $q_{n}$ to represent Optical Transfer Functions (OTF) characterized by including the diffraction-limited OTF as the first basis function $q_{0}=\mathrm{OTF}_{\text {perfect }}$. To this end we apply a powerful and rigorous theoretical framework based on applying the appropriate change of variables to well-known orthogonal systems [2]. Here we depart form spherical harmonics or Legendre polynomials for the particular case of rotational-symmetry. Numerical experiments with different examples show that the number of terms necessary to obtain an accurate linear expansion of the OTF mainly depends on the image quality. In the rotationally symmetric case we obtained a reasonable accuracy with around $\mathbf{1 0}$ basis functions, but in general for cases of poor image quality the number of basis functions may increase and hence affect the efficiency of the method. Other potential applications, such as new image quality metrics are also discussed. () 2016 Optical Society of America
\end{abstract}

OCIS codes: (110.4850) Optical transfer functions; (000.4430) Numerical approximation and analysis; (110.3925) Metrics; (220.4840) Optical testing.

http://dx.doi.org/10.1364/ao.XX.XXXXXX

\section{INTRODUCTION}

The use of orthogonal basis to describe surfaces and wavefronts has shown to be crucial in optical science and technology. Zernike polynomials [8] have become the standard for representing wave aberrations in atmospheric optics [11], visual optics (ANSI Z80.28 standard for reporting aberrations in the human eye), optical design and testing [8], etc. Zernike polynomials were also used for shape specification of optical surfaces (aspheres, free-form, etc.) [9]. In the case of optical surfaces, it may be of practical interest to use a specific system to describe the departure from the sphere, since the sphere is the most widely used optical surface, and often many designers start from spheres (or conicoids) for preliminary paraxial computations. In this sense Forbes proposed a highly successful set of functions, adapted from orthogonal polynomials, to specify aspheres [3] and free-form [4] surfaces. In a recent work we proposed a method to generate systems in which the first basis function is the sphere (or conicoid) and the rest of functions are orthogonal to it [2]. Complex Zernike polynomials were also used in the extended Nijboer-Zernike theory for the computation of optical Point-Spread Functions (PSF) [5], as they can represent the complex pupil function that is both the amplitude and phase of a wavefront at the (circular) pupil plane [10]. Optical image quality is fully determined by the PSF or alternatively by its Fourier transform, the Optical Transfer Function (OTF). It is common to measure either the PSF or the OTF (and often only its modulus, the MTF, is available), but then the wavefront cannot be retrieved in general. Therefore, a compact description of the wavefront as a set of Zernike or similar coefficients is not available in these cases. Our purpose here is to find an orthogonal basis to describe the complex two-dimensional OTF in terms of a set of coefficients $c_{n}$ of the corresponding linear expansion on the basis functions, in a similar way as surfaces, wavefronts, etc.:

$$
\operatorname{OTF}(r, \theta)=\sum_{n=1}^{\infty} c_{n} q_{n}(r, \theta),
$$

where $r$ represents normalized spatial frequencies, with $0 \leq r \leq$ 1 in polar coordinates. This means that the OTF takes values on a unit circle. This is a crucial property, which is not applicable to the PSF, as the extent of the PSF gets wider as the optical quality is worse. It is noteworthy to mention that Zernike polynomials or similar basis are not adequate for representing typical OTFs having a sharp tip at the origin with a lack of continuity in its derivative. Even using a 12th-order Zernike polynomial expansion is not possible to accurately fit that tip (see Fig. 1 in Ref. [13]). A totally different approach was proposed by Schwiegerling [13], who applied the method of Kintner and Sillitto [6] to abtain a linear expansion of the OTF in terms of the wavefront coefficients. This formulation was later extended to the general non radially symmetric case [14]. Even 
though this approach was recently improved by using the Meijer G-functions [1], the resulting linear expansion has important drawbacks. The Meijer G-functions are not orthogonal, and the method is not computationally efficient [1].

For this reason, here we apply the method that we developed in our previous work [2], which basically consists of (1) departing from an initial function of the basis $q_{0}(r)$, and (2) obtaining a complete system of functions orthogonal to it (and between them) by the appropriated change of variables applied to a given orthogonal system (polynomials typically). Here we chose the OTF of the perfect (diffraction-limited) optical system as the first basis function, using its analytical expression:

$$
q_{0}(r)=\mathrm{OTF}_{\text {perfect }}(r)=\frac{2}{\pi}\left[\arccos (r)-r \sqrt{1-r^{2}}\right] .
$$

Therefore our goal is to obtain a linear expansion of the OTF in terms of a complete orthogonal basis, at the cost of missing a explicit relationship of the expansion coefficients of the OTF with those of the wavefront.

We consider first the case of rotational symmetry $\operatorname{OTF}(r)$, which is a common situation in many optical systems working on-axis. Then we extend the basis to the general twodimensional case. The resulting basis, both rotationally symmetric and general, are tested by least squares fitting on different examples.

\section{BASIS FOR ROTATIONALLY SYMMETRIC OTFS}

In the first part of this section we briefly summarize the general theory introduced in [2], restricted to rotationally symmetric OTFs defined by an equation of the form $f=f(r)$, where $r \in[0,1]$ is the normalized radial frequency: we design an orthogonal system for $L_{v}^{2}[0,1]$ with measure $d v=r d r$, in which the first element of the system is a specified function that we denote by $q_{0}(r)$ for convenience; the remaining elements of the system are denoted by $q_{n}(r), n=1,2,3, \ldots$. In the second part of this section we apply the theory to the particular case in which the orthogonal system is derived from the set of Legendre polynomials and $q_{0}(r)$ is the diffraction-limited $\mathrm{OTF}_{\text {perfect }}$.

\section{A. General theory}

Let $\left\{p_{n}(x)\right\}_{n=0,1,2, \ldots}$ be an orthonormal basis of $L_{\mu}^{2}[c, d]$ with $p_{0}(x)=p_{0}$ constant and measure $d \mu=\rho(x) d x$. This means that

$$
\delta_{m, n}=\int_{c}^{d} p_{n}(x) p_{m}(x) \rho(x) d x, n, m=0,1,2, \ldots
$$

We define, for $n=0,1,2, \ldots$, the functions

$$
q_{n}(r):=\frac{C_{n}}{C_{0} p_{0}} q_{0}(r) p_{n}(\varphi(r)), n=0,1,2, \ldots,
$$

where $C_{n}:=1$ for $n=1,2,3, \ldots$,

$$
C_{0}^{2}:=\int_{0}^{1} s q_{0}^{2}(s) d s /\left[p_{0}^{2} \int_{c}^{d} \rho(t) d t\right]
$$

and the function $\varphi(r)$ is implicitly defined by the equation

$$
\int_{c}^{x} \rho(t) d t=\frac{1}{C_{0}^{2} p_{0}^{2}} \int_{0}^{r} s q_{0}^{2}(s) d s, x=\varphi(r) .
$$

It is shown in [2] that the set $\left\{q_{n}(r)\right\}_{n=0,1,2, \ldots}$ is a quasiorthonormal basis of $L_{v}^{2}[0,1]$ (orthonormal except for the fact that $\left.\left\|q_{0}\right\|_{r}^{2}=C_{0}^{2}\right)$. Moreover, $\left\{q_{n}(r)\right\}_{n=0,1,2, \ldots}$ is complete in $L_{v}^{2}[0,1]$; that is, for any function $F(r) \in L_{v}^{2}[0,1]$, we have

$$
F(r)=\frac{c_{0}}{C_{0}} q_{0}(r)+\sum_{n=1}^{\infty} c_{n} q_{n}(r),
$$

where the coefficients are given by the projections on the basis functions:

$$
c_{n}:=\frac{1}{C_{n}} \int_{0}^{1} q_{n}(r) F(r) r d r, \quad n=0,1,2,3, \ldots
$$

The equality in Eq. (7) is understood in the $L_{v}^{2}$ sense, and also pointwise when $F(r)$ is a continuous function in $[0,1]$.

\section{B. Solution based on Legendre polynomials}

In the remaining of this section we consider the following particularly important example: the set $\left\{p_{n}(x)\right\}_{n=0,1,2, \ldots}$ is the set of normalized Legendre polynomials [15]:

$p_{n}(x)=\sqrt{\frac{2 n+1}{2}} \frac{1}{2^{n}} \sum_{k=0}^{n}\left(\begin{array}{l}n \\ k\end{array}\right)^{2}(x-1)^{n-k}(x+1)^{k}, p_{0}(x)=\frac{1}{\sqrt{2}}$.

It is an orthonormal basis of $L^{2}[-1,1]$ with respect to the weight function $\rho(x)=1$. We take OTF perfect $_{\text {as }}$ as the first basis function:

$$
q_{0}(r)=\frac{2}{\pi}\left[\arccos (r)-r \sqrt{1-r^{2}}\right]
$$

with $r \equiv \cos \alpha, 0 \leq r \leq 1,0 \leq \alpha \leq \frac{\pi}{2}$. We compute the change of variable $\varphi(r)$ from Eq. (6):

$$
\begin{aligned}
x+1= & \int_{-1}^{x} d t=\frac{8}{C_{0}^{2} \pi^{2}} \int_{0}^{r} t\left[\arccos (t)-t \sqrt{1-t^{2}}\right]^{2} d t \\
= & \frac{1}{12 \pi^{2} C_{0}^{2}}\left[3 \pi^{2}-4 r^{2}\left(3-3 r^{2}+4 r^{4}\right)\right. \\
& \left.-24 r\left(1+2 r^{2}\right) \sqrt{1-r^{2}} \arccos (r)+12\left(4 r^{2}-1\right) \arccos ^{2}(r)\right] .
\end{aligned}
$$

We obtain the value of $C_{0}$ from Eq. (5),

$$
C_{0}=\sqrt{\frac{1}{8}-\frac{2}{3 \pi^{2}}} .
$$

Thus,

$$
\begin{aligned}
x=\varphi(r)= & \frac{1}{3 \pi^{2}-16}\left[3 \pi^{2}+16-8 r^{2}\left(3-3 r^{2}+4 r^{4}\right)\right. \\
& -48 r\left(1+2 r^{2}\right) \sqrt{1-r^{2}} \arccos (r) \\
& \left.+24\left(4 r^{2}-1\right) \arccos ^{2}(r)\right] .
\end{aligned}
$$

Therefore, we have that the set

$$
q_{n}(r)=\frac{8 \sqrt{3}}{\sqrt{3 \pi^{2}-16}} C_{n}\left(\arccos (r)-r \sqrt{1-r^{2}}\right) p_{n}(\varphi(r)),
$$

for $n=0,1,2, \ldots$, is a quasi-orthonormal basis of $L_{v}^{2}[0,1]$ with $d v=r d r$ or of $L_{\bar{v}}^{2}[0, \pi / 2]$ with $d \bar{v}=\sin \alpha \cos \alpha d \alpha$ and any function $F(r) \in L_{v}^{2}[0,1]$, and in particular any OTF can be written in the form

$$
f(r)=\frac{c_{0}}{C_{0}} \frac{2}{\pi}\left[\arccos (r)-r \sqrt{1-r^{2}}\right]+\sum_{n=1}^{\infty} c_{n} q_{n}(r),
$$




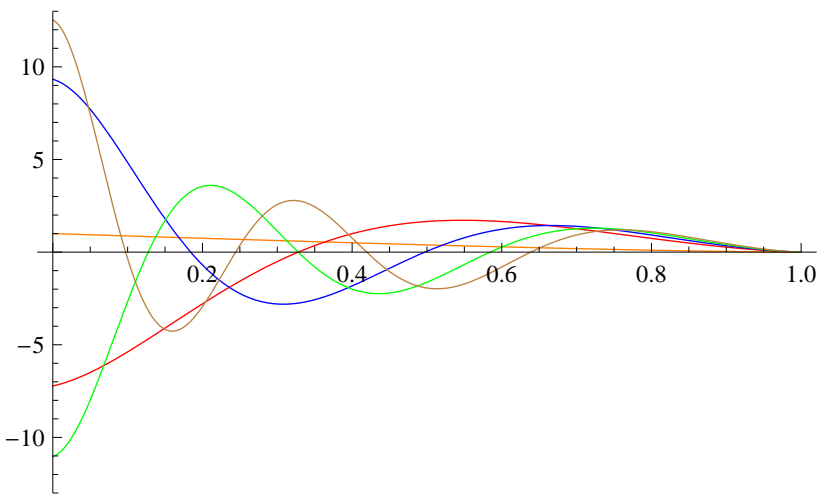

Fig. 1. Graphs of the first five functions of the new quasi-orthonormal basis $\left\{q_{n}(r)\right\}_{n=0,1,2, \ldots}$ (see Eq. (14)) obtained from the normalized Legendre polynomials and $q_{0}(r)=\frac{2}{\pi}\left[\arccos (r)-r \sqrt{1-r^{2}}\right]$ using different scale: $q_{0}$ (orange), $q_{1}$ (red), $q_{2}$ (blue), $q_{3}$ (green), $q_{4}$ (brown).

with $c_{n}, C_{0}$ and $q_{n}(r)$ given in Eq. (8), Eq. (12) and Eq. (14) respectively.

The graphs of the first five functions $q_{0}(r), q_{1}(r), \ldots, q_{4}(r)$ are illustrated in Fig. 1.

The theory developed in this section only applies to rotationally symmetric functions, and in particular OTFs specified by an equation of the form $f=f(r)$, with $r \in[0,1]$. In the following section we generalize the theory to the general two-dimensional case, specified by an equation of the form $f=f(r, \theta)$.

\section{BASIS FOR GENERAL TWO-DIMENSIONAL OTFS}

In the first part of this section we briefly summarize the more general theory introduced in [2] to approximate arbitrary functions $f=f(r, \theta)$ defined over the unit disk $\mathrm{D},(r \cos \theta, r \sin \theta) \in$ $D$ : we design an orthogonal system for $L_{v}^{2}(D)$ with measure $d v=r d r d \theta$ in which the first element of the system is a specified rotationally symmetric function $q_{0}^{0}(r, \theta)=q_{0}^{0}(r)$. The remaining elements of the system $q_{n}^{m}(r, \theta), n=1,2,3, \ldots$, are constructed using again the three essential ingredients used in the previous section: (i) an arbitrary orthogonal system $p_{n}^{m}(x, \theta), n, m(n)=$ $0,1,2, \ldots$, of $L_{\mu}^{2}([c, d] \times[0,2 \pi])$ with measure $d \mu=\rho(x) d x d \theta$, (ii) the first element of the system $q_{0}^{0}(r)$ and (iii) a convenient change of variable $x=\varphi(r), \varphi:[0,1] \rightarrow[c, d]$. Then, the resulting orthogonal system consists of functions $\left\{q_{0}^{0}(r), q_{m}^{n}(r, \theta), \ldots\right\}$, $n, m(n)=1,2, \ldots$, defined in the unit disk $D$ that are orthogonal with respect to the measure $d v=r d r d \theta$. Moreover, the functions $q_{m}^{n}(r, \theta), \ldots, n, m(n)=1,2, \ldots$ are also orthonormal. In the second part of this section we give the particularly important example in which the $q_{m}^{n}(r, \theta)$ functions are obtained from the spherical harmonics and $q_{0}(r)$ is the diffraction-limited $\mathrm{OTF}_{\text {perfect }}$ function.

Let $\left\{p_{n}^{m}(x, \theta)\right\}_{n, m(n)=0,1,2, \ldots}$ be an orthonormal basis of $L_{\mu}^{2}([c, d] \times[0,2 \pi])$ with $p_{0}^{0}(x, \theta)=p_{0}^{0}$ constant and measure $d \mu=\rho(x) d x d \theta$. We want to find a quasi-orthonormal basis $\left\{q_{n}^{m}(r, \theta)\right\}_{n, m(n)=0,1,2, \ldots}$ of $L_{v}^{2}(D), d v=r d r d \theta$, with $q_{0}^{0}(r)$ predetermined. We have

$$
\delta_{n, n^{\prime}} \delta_{m, m^{\prime}}=\int_{c}^{d} \rho(x) d x \int_{0}^{2 \pi} d \theta p_{n}^{m}(x, \theta) p_{n^{\prime}}^{m^{\prime}}(x, \theta),
$$

for $n, m(n)=0,1,2, \ldots$ We define, for $n, m(n)=0,1,2, \ldots$, the functions

$$
q_{n}^{m}(r, \theta)=\frac{C_{n}^{m}}{C_{0}^{0} p_{0}^{0}} q_{0}^{0}(r) p_{n}^{m}(\varphi(r), \theta),
$$

where $C_{n}^{m}:=1$ for $(n, m) \neq(0,0)$,

$$
\left(C_{0}^{0}\right)^{2}:=\int_{0}^{1} s\left(q_{0}^{0}(s)\right)^{2} d s /\left[\left(p_{0}^{0}\right)^{2} \int_{c}^{d} \rho(t) d t\right],
$$

and the function $\varphi(r)$ is implicitly defined in Eq. (6) with $C_{0}$ replaced by $C_{0}^{0}, p_{0}$ by $p_{0}^{0}$ and $q_{0}(r)$ by $q_{0}^{0}(r)$. Then, we have that the set of functions $\left\{q_{n}^{m}(r, \theta)\right\}_{n, m(n)=0,1,2, \ldots}$ is a quasi-orthonormal system of $L_{v}^{2}(D)$. Moreover, the system $\left\{q_{n}^{m}(r, \theta)\right\}_{n, m(n)=0,1,2, \ldots}$ is complete in $L_{v}^{2}(D)$; for any $F(r, \theta) \in$ $L_{v}^{2}(D)$, we have that

$$
F(r, \theta)=\frac{c_{0}^{0}}{C_{0}^{0}} q_{0}^{0}(r)+\sum_{\substack{n, m(n)=0 \\(n, m) \neq(0,0)}}^{\infty} c_{n}^{m} q_{n}^{m}(r, \theta),
$$

with

$$
c_{n}^{m}:=\frac{1}{C_{n}^{m}} \iint_{D} q_{n}^{m}(r, \theta) F(r, \theta) r d r d \theta, \quad n, m(n)=0,1,2,3, \ldots
$$

The equality in Eq. (19) is understood in the $L_{v}^{2}$ sense, and also pointwise when $F(r, \theta)$ is a continuous function in $D$.

We consider now the following particularly important example: the set $\left\{p_{n}^{m}(x, \theta)\right\}_{n, m(n)=0,1,2, \ldots}$ is the set of spherical harmonic functions [16]:

$$
\begin{aligned}
p_{n}^{m}(x, \theta)= & \sqrt{\frac{\left(2-\delta_{m, 0}\right)(2 n+1)(n-m) !}{4 \pi(n+m) !}} \\
& \times \begin{cases}P_{n}^{m}(x) \cos (m \theta), & 0 \leq m \leq n, \\
P_{n}^{m}(x) \sin (m \theta), & -n \leq m<0,\end{cases}
\end{aligned}
$$

where $P_{n}^{n}(x)$ are the associated Legendre polynomials [17]:

$P_{n}^{m}(x)=\frac{(-1)^{m}}{2^{n} n !}\left(1-x^{2}\right)^{m / 2} \frac{d^{n+m}}{d x^{n+m}}\left(x^{2}-1\right)^{n}, p_{0}^{0}(x, \theta)=\frac{1}{2 \sqrt{\pi}}$.

They are an orthonormal basis of $L_{\mu}^{2}([-1,1] \times[0,2 \pi])$ with measure $d \mu=d x d \theta$. We choose $q_{0}^{0}(r)$ to be the diffraction-limited OTF as a first approximation of the OTF. Then, from Eq. (6) with $C_{0}$ replaced by $C_{0}^{0}, p_{0}$ by $p_{0}^{0}$ and $q_{0}(r)$ by $q_{0}^{0}(r)$, and using the value of $C_{0}^{0}$ obtained from Eq. (18), we derive the same $\varphi(r)$ given in Eq. (13) for the rotationally symmetric case. Therefore, the set $\left\{q_{n}^{m}(r, \theta)\right\}_{n=0,1,2, \ldots}^{m=-n, \ldots, n}$, with

$$
q_{n}^{m}(r, \theta)=\frac{8 \sqrt{3} C_{n}}{\sqrt{3 \pi^{2}-16}}\left(\arccos (r)-r \sqrt{1-r^{2}}\right) p_{n}^{m}(\varphi(r), \theta)
$$

is a quasi-orthonormal basis of $L_{v}^{2}(D)$ with $d v=r d r d \theta$. In particular, we have that $q_{0}^{0}(r, \theta)=\frac{2}{\pi}\left[\arccos (r)-r \sqrt{1-r^{2}}\right]$.

Fig. 2 shows the absolute value of first functions (up to $n=$ 4 , and $m \geq 0)$ for $r \in[0,1], \theta \in[0,2 \pi]$.

It is important to note that the OTF is complex-valued in general, whereas the basis functions obtained from the spherical harmonics are real. However, the OTF is a linear combination of the spherical harmonics with constant coefficients $c_{n}^{m}$ and then, these coefficients $c_{n}^{m}$ must be complex numbers: from Eq. (20) we see that they are computed as integrals of the OTF function multiplied by spherical harmonics and then, in general, they are complex numbers. 


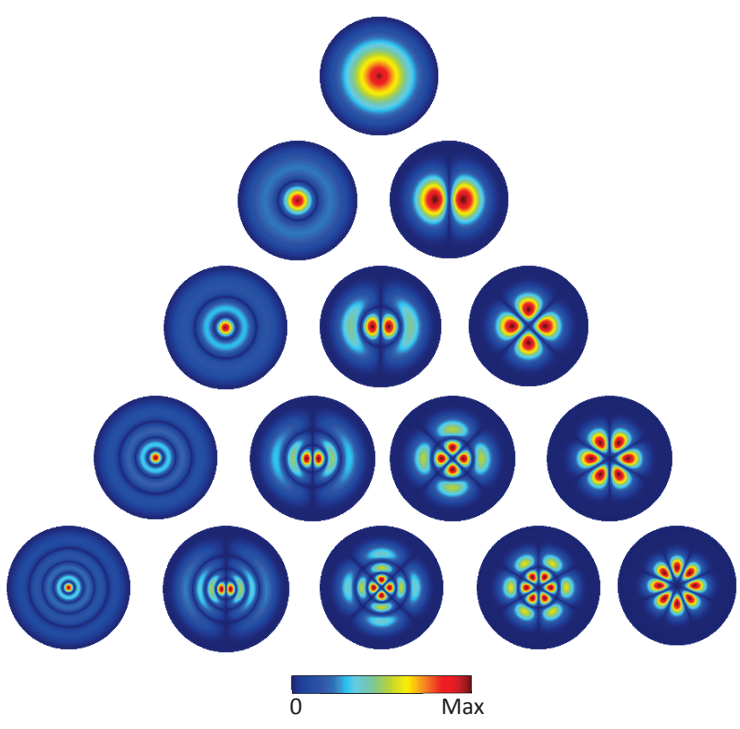

Fig. 2. Absolute value of the first functions $q_{n}^{m}(r, \theta)$ (see Eq. (23)) for the case of the spherical harmonics and $q_{0}^{0}(r, \theta)=$ $\frac{2}{\pi}\left[\arccos (r)-r \sqrt{1-r^{2}}\right]$. The rows represent the ascending order from $n=0$ to $n=4$, the columns are the positive values of $m$ from $m=0$ to $m=n$.

\section{IMPLEMENTATION AND EXAMPLES}

For the numerical implementation and testing of the new basis proposed in Sections 2 and 3, we consider different examples of both rotationally-symmetric (1D) and general (2D) cases.

\section{A. Rotationally symmetric OTFs}

In order to check the accuracy of the approximation supplied by the basis $\left\{q_{n}(r)\right\} n=0,1,2, \ldots$ in the rotationally symmetric case given in Eq. (14), we consider two examples of real OTFs. Each $f(r)$ is uniformly sampled in 0.01 steps which yields 101 points in the interval $[0,1]$. The first example is the OTF computed from a wave aberration that is the sum of three non-zero Zernike coefficients: $c z_{2}^{0}=\lambda / 5$ (defocus), $c z_{4}^{0}=-\lambda / 10$ (4th order spherical aberration), and $c z_{6}^{0}=\lambda / 15$ (6th order spherical aberration), where $\lambda$ is the wavelengh; the second example corresponds to another combination of the same aberrations, but with higher values: $c z_{2}^{0}=\lambda / 2, c z_{4}^{0}=-\lambda / 4, c z_{6}^{0}=\lambda / 8$.

Fig. 3 shows the different approximations of both OTFs (left and right panels respectively), obtained as we increase the number of basis functions. The plots represent absolute values (MTFs), that is modulation versus spatial frequency $r$. The coefficients of the last approximation (for $n=10$ ) are given in Table 1. The RMS fit errors are also included in the figure legend.

We can observe that the magnitudes of the expansion coefficients tend to decrease rapidly with $n$, which ensures a reasonable convergence. Nevertheless, as the OTF becomes worse (second example) then the first coefficient (corresponding to the perfect OTF) is lower, whereas the rest of coefficients are higher. The second OTF presents not only lower values, but it looks more wavy, which means that we need more (higher order) basis functions for an accurate reconstruction. The worse the image quality, the higher number of basis functions are needed. This also suggests the possibility of using the expansion coeffi-
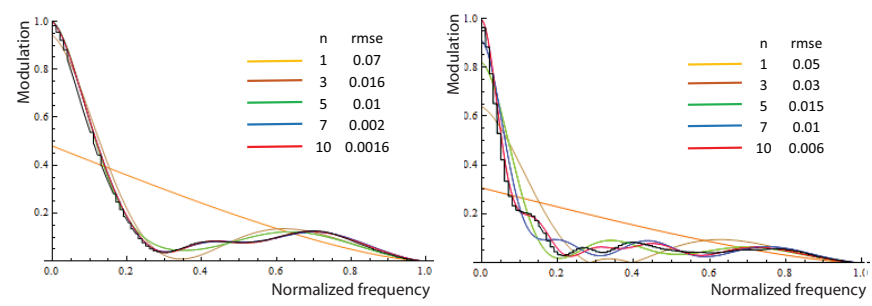

Fig. 3. Reconstructions obtained for different ascending values of $n$. The left graph corresponds to the first OTF, the right to the second OTF. For each approximation we show the root mean square fit error (rmse).

\begin{tabular}{|c|c|c|c|c|}
\hline$c_{0}$ & $c_{1}$ & $c_{2}$ & $c_{3}$ & $c_{4}$ \\
0.29129 & -0.012629 & 0.05293 & -0.003195 & 0.010919 \\
0.16498 & -0.003885 & 0.022527 & -0.009078 & 0.013237 \\
$c_{5}$ & $c_{6}$ & $c_{7}$ & $c_{8}$ & $c_{9}$ \\
0.008416 & 0.004399 & -0.00087 & -0.001057 & -0.000256 \\
0.0026327 & 0.0081033 & -0.002348 & 0.005106 & -0.0063212 \\
\hline
\end{tabular}

Table 1. Expansion coefficients obtained for $n=10$. The first row corresponds to the first OTF and the second row to the second OTF.

cients to build new image quality metrics, which is discussed in the next Section.

\section{B. General case: 2D OTFs}

In order to check the accuracy of the approximation supplied by the basis $\left\{q_{n}^{m}(r, \theta)\right\} \begin{aligned} & m=-n, \ldots, n \\ & n=0,1,2, \ldots\end{aligned}$ in the general case given in Eq. (23), we consider one example taken from a data base of OTFs measured in human eyes for a $5 \mathrm{~mm}$ pupil diameter [12]. We want to remark that this is a particularly difficult and interesting example, since human eyes present all kinds of aberration modes (Zernike coefficients), so the associated OTF tends to be far from the perfect system $q_{0}^{0}(r)$, and far from the rotational symmetry. Then these OTFs are complex-valued and typically show irregular and complicated patterns, as we can see in the example shown in the lower right panel in Fig. 4.

This OTF was given as a $201 \times 201$ matrix of complex numbers, corresponding to a sampling interval of 0.01 , between -1 and 1 (normalized frequency). The central point at zero frequency is at $(101 ; 101)$. Fig. 4 shows different reconstructions which improve as we increase the radial order $n$. For these two-dimensional poor image quality OTFs, we needed a high number of basis functions (up to 256 for $n=15$ ), to obtain a reasonable approximation, which may become impractical for low-quality OTFs.

\section{DISCUSSION}

So far we presented orthogonal systems for representing both rotationally symmetric (one-dimensional) and general (twodimensional) Optical Transfer Functions. The way to find a system adapted to this particular type of functions was to build it starting from the diffraction-limited $q_{0}=\mathrm{OTF}_{\text {perfect }}$ as the first basis function of the system. The fact that $q_{0}$ has a reasonable compact analytical expression enables the implementation of this idea. The rest of the basis functions $\left\{q_{n}\right\}_{n=0,1,2, \ldots}$ are then obtained by applying a general, rigorous and powerful frame- 

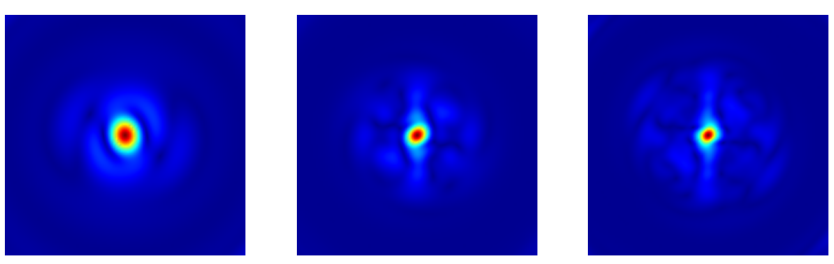

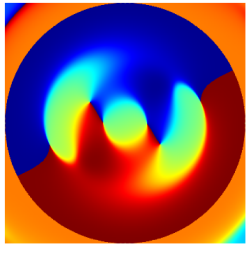

$(3,0.0227)$
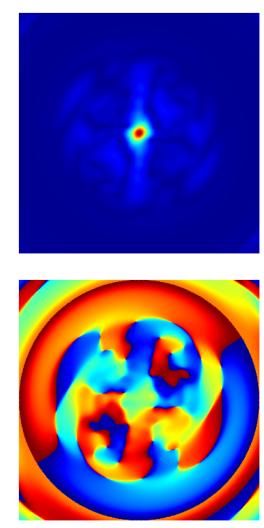

$(13,0.0155)$

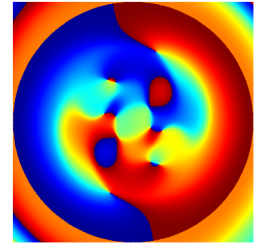

$(8,0.018)$
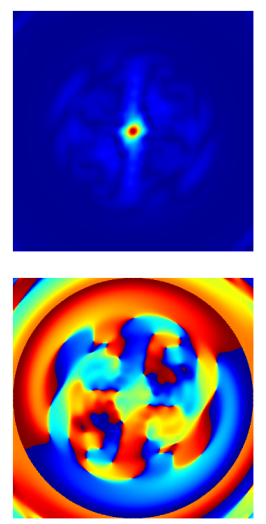

$(15,0.0146)$

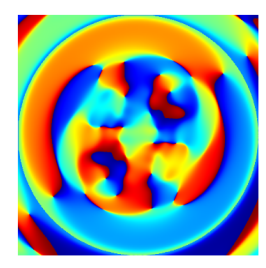

$(11,0.016)$
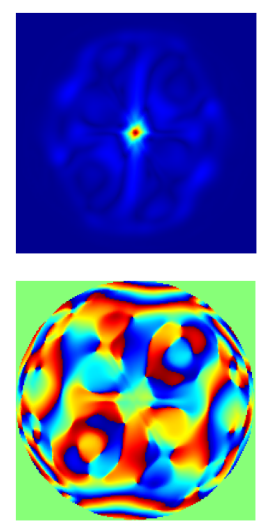

original
Fig. 4. Density plots of the absolute value (MTF) and the phase function of the reconstructions given by formula 4 for different ascending values of $n$. For each approximation we show the rms error below (in parenthesis (n, rmse)). The last density plot corresponds to the reading of the data base.

work to obtain orthogonal systems, that we developed before [2] for the particular case of representing aspheric and free-form optical surfaces. That method consists of finding the appropriate change of variables transforming a well-known orthogonal system (Legendre polynomials and spherical harmonics were used here for 1D and 2D respectively) into the desired new system. The necessary condition for that all the basis functions have analytical expressions is that the integral equation Eq. (6) has an analytical solution, which was indeed the case for our choice $q_{0}=\mathrm{OTF}_{\text {perfect }}$.

Therefore the main advantage of the proposed OTF-specific systems is to have a general analytical expression, in the form of the classical linear expansion in terms of orthogonal basis functions. Particular OTFs are then represented by a set of coefficients, which are given by the projections of the OTF on the different basis functions. This type of analytical representation may be interesting in a variety of potential applications. In practice, it is desirable to have a representation as compact as possible. In this sense, our results with different examples suggest that the representation may be reasonably compact either in the rotationally symmetric case or in the general case when the OTF is not too far from $q_{0}^{0}=\mathrm{OTF}_{\text {perfect }}$. For non-rotationally sy- mmetric strongly aberrated optical systems the corresponding OTF is far away from $q_{0}^{0}$ and then the number of non-zero coefficients in the expansion could be too high to be of practical use. In those cases, with poor OTFs, one has to choose the accuracy of the approximation required for the particular application. The classic problem of relating the expansion coefficients of the OTF with those of the wave aberration $W$ (typically given as a Zernike polynomial expansion) remains unsolved in general, especially for large aberration coefficients. The problem is that there are two nonlinearities involved in computing the incoherent OTF from the wave aberration. The first one is the complex exponentiation (strongly non-linear) associated to the fact that $W$ is the phase of the complex pupil function. The second one is the squared modulus associated to incoherent OTF. These two nonlinearities preclude to retrieve $W$ from the OTF. In particular, the inversion of the squared modulus is the wellknown ill-posed phase retrieval problem [7] , which is outside the scope of this work. On the other hand attempts to overcome these nonlinearities [13], are limited to low values of aberrations, and are far from being computationally efficient [1].

We believe that another interesting application is to define new image quality metrics. One of the most popular metric is the volume of the OTF normalized by the volume of the $\mathrm{OTF}_{\text {perfect }}$; i.e. the Strehl ratio:

$$
\mu_{1}:=\frac{\int_{0}^{2 \pi} \int_{0}^{1} f(r) r d r d \theta}{\int_{0}^{2 \pi} \int_{0}^{1} q_{0}(r) r d r d \theta}=\frac{c_{0}}{C_{0}}+\frac{1}{P} \sum_{n=1}^{\infty} c_{n} \int_{0}^{2 \pi} \int_{0}^{1} q_{n}(r) r d r d \theta
$$

in the one-dimensional case, or

$$
\begin{aligned}
\mu_{1} & :=\frac{\int_{0}^{2 \pi} \int_{0}^{1} f(r, \theta) r d r d \theta}{\int_{0}^{2 \pi} \int_{0}^{1} q_{0}^{0}(r, \theta) r d r d \theta} \\
& =\frac{c_{0}^{0}}{C_{0}^{0}}+\frac{1}{P} \sum_{(n, m) \neq(0,0)}^{\infty} c_{n}^{m} \int_{0}^{2 \pi} \int_{0}^{1} q_{n}^{m}(r, \theta) r d r d \theta
\end{aligned}
$$

in the two-dimensional case, where $P=\int_{0}^{2 \pi} \int_{0}^{1} q_{0}(r) r d r d \theta$ or $\int_{0}^{2 \pi} \int_{0}^{1} q_{0}^{0}(r, \theta) r d r d \theta$ respectively. Observe that once we have computed the integrals of the elements $q_{n}$ or $q_{n}^{m}$ of the basis, we may compute $\mu_{1}$ for any OTF by means of linear combination of coefficients.

Other possible image quality metric that measures the proximity of $f(r)$ to $q_{0}(r)$ or $f(r, \theta)$ to $q_{0}(r, \theta)$ is the coefficient of $q_{0}(r)$ (or $q_{0}(r, \theta)$ ) in the orthonormal expansion of the function $f(r)$ or $f(r, \theta)$ :

$$
\mu_{2}=\frac{c_{0}}{C_{0}}, \quad \text { or } \quad \mu_{2}=\frac{c_{0}^{0}}{C_{0}^{0}}
$$

Finally, another possible image quality metrics is analogous to the $\mu_{1}$ metric, but replacing the $L_{1}$ norms of the functions by their $L_{2}$ norms:

$$
\mu_{3}=\frac{1}{C_{0}} \sqrt{\sum_{n=0}^{\infty}\left|c_{n}\right|^{2}}, \quad \text { or } \quad \mu_{3}=\frac{1}{C_{0}^{0}} \sqrt{\sum_{n, m(n)=0}^{\infty}\left|c_{n}^{m}\right|^{2}} .
$$

These three metrics are normalized having values within the interval $[0,1]$, and they reach their maximum values (1) for $\mathrm{OTF}_{\text {perfect. }}$. The values obtained for the above examples are listed in Table 1. The three metrics give similar values for the rotationally symmetric examples (4.1.1 and 4.1.2), whereas they show quite different values for the poor two-dimensional 


\begin{tabular}{c|c|c|c} 
& $\mu_{1}$ & $\mu_{2}$ & $\mu_{3}$ \\
\hline Ex 4.1.1 & 0.349 & 0.291 & 0.374 \\
\hline Ex 4.1.2 & 0.194 & 0.165 & 0.208 \\
\hline Ex 4.2 & 0.009 & 0.031 & 0.186 \\
\hline
\end{tabular}

Table 2. Values of the four image quality metrics given for the different examples.

complex OTF. In this case $\mu_{2}$ provides an adequate quantitative grading of the OTF quality. It is also the simplest metric given by the first coefficient $\left(q_{0}\right)$ of the expansion.

In summary, we believe that this type of modal representation based on analytical and orthogonal modes (basis functions) may be of interest in different aspects, including the possibilities of physical interpretation of the OTF in terms of the values (coefficients) of these modes.

\section{FUNDING INFORMATION}

Spanish Ministry of Economía y Competitividad (MTM201452859) and the European Union (FIS2014-58303).

\section{REFERENCES}

1. J. A. Díaz, "Relating wavefront error, apodization, and the optical transfer function: Comment", JOSA A, in press (2016).

2. C. Ferreira, J. L. López, R. Navarro, E. Pérez Sinusía, "Orthogonal basis with a conicoid first mode for shape specification of optical surfaces", Opt. Express 24, 5448-5462 (2016).

3. G. W. Forbes, "Shape specification for axially symmetric optical surfaces", Opt. Express 15(8), 5218-5226 (2007).

4. G. W. Forbes, "Fitting freeform shapes with orthogonal bases", Opt. Express 21(16), 19061-19081 (2013).

5. A. J. Janssen, "Extended Nijboer-Zernike approach for the computation of optical point spread functions", JOSA A, 19(5), 849-857 (2002).

6. E. C. Kintner and R. M. Sillitto, "A new 'analytic' method for computing the optical transfer function", Opt. Acta 23, 607-619 (1976).

7. M. V. Klibanov, P. E. Sacks, A.V. Tikhonravov, "The phase retrieval problem", Inverse Problems 11 1--28 (1995).

8. V. N. Mahajan, Zernike polynomials and wavefront fitting, in Optical Shop Testing, 3rd ed., D. Malacara, ed. Wiley, New York, (2007).

9. J. Nam, and J. Rubinstein, "Numerical reconstruction of optical surfaces", JOSA A, 25(7), 1697-1709 (2008).

10. R. Navarro, R. Rivera and J. Aporta, "Representation of wavefronts in free-form transmission pupils with Complex Zernike Polynomials", J Optom., 4(2), 41-48 (2011).

11. R. J. Noll,"Zernike polynomials and atmospheric turbulence", J. Opt. Soc. Am. 66(3), 207-211 (1976).

12. J. J. Rozema, P. Rodriguez, R. Navarro, M. J. Tassignon, "SyntEyes: a Higher Order Statistical Eye Model for Healthy Eyes", Invest. Ophth. Vis. Sci. 57, 683-691 (2016).

13. J. Schwiegerling, "Relating wavefront error, apodization, and the optical transfer function: on-axis case", JOSA A, 31, 2476-2483 (2014).

14. J. Schwiegerling, "Optical transfer function optimization based on linear expansions," Novel Optical Systems Design and Optimization XVIII, Proc. SPIE 9579, 95790H, (2015).

15. E. W. Weisstein, "Legendre Polynomial", From MathWorld. A Wolfram Web Resource. http://mathworld.wolfram.com/LegendrePolynomial.html.

16. E. W. Weisstein, "Spherical Harmonic", From MathWorld. A Wolfram Web Resource. http://mathworld. wolfram.com/SphericalHarmonic.html.

17. E. W. Weisstein, "Associated Legendre Polynomial", From MathWorld. A Wolfram Web Resource. http://mathworld.wolfram.com/AssociatedLegendrePolynomial.html. 